\title{
A PLANETARIUM ORIENTED SEQUENCE OF EXERCISES
}

\author{
Carlson R. Chambliss \\ Kutztown University, Kutztown, Pennsylvania 19530, U.S.A.
}

The introductory course in astronomy intended for non-majors is taken by about 150 students per semester at Kutztown University. This institution is fortunate in having a planetarium, which is used extensively both in course work in astronomy and with visiting school groups. The projector is a Spitz model A3P, the dome is of perforated aluminum and has a diameter of 10.5 meters, and the seating capacity of the planetarium is about 120 . The introductory astronomy course at Kutztown consists of two hours of lectures and a two-hour laboratory session per week. There are five or six laboratory sections, each of which contains about 25-30 students.

The planetarium has proven to be extremely useful in demonstrating numerous concepts of the celestial sphere. At Kutztown University, a three-part sequence of laboratory exercises on the celestial sphere is required near the beginning of the introductory course. In the first week of this sequence the students are introduced to the altazimuth system and to such terms as cardinal points, celestial poles, meridian, equator, and great circles. All of these can be demonstrated with the illuminated circles and markings that are provided in the planetarium. The relationship between the altitude of the north celestial pole and of its compliment (the inclination of the celestial equator to the horizon) to the latitude of the observer is stressed.

In the following unit, such concepts as hour angle and circumpolar stars are introduced together with the annual motion of the sun and the equatorial coordinate system. The appearance of the night sky is shown at a wide variety of latitudes, and the role of astronomical observations in determining one's position on the Earth's surface is discussed. The relationship between the altazimuth and equatorial coordinate systems is examined, although the trigonometric equations that allow for the transformation of positions from one system to the other are not used in this introductory course. Various stars and constellations are pointed out, and later on in the course students are given a quiz in which they are required to identify various bright stars and prominent constellations.

In the third and final part of the exercises on the celestial sphere the seasons are discussed together with ecliptic coordinates, precession of the equinoxes, and a brief introduction to climatology. Throughout the sequence, students are required to make estimates of various parameters from observations that they make on the planetarium dome.

The conceptualization of the celestial sphere involves many terms that are initially unfamiliar to the non-science student, but by breaking up this study into three units, usually spread over a period of three weeks, we find that nearly all students can successfully grasp the terminology and the interrelations among the 
various parameters of the celestial sphere.

A question arises as to how much material of this sort should be included in one-semester course for non-science majors. Formerly the equatorial coordinates of the north and south poles of the ecliptic were derived from diagrams, but we have concluded that this is superfluous to the syllabus of this course. Galactic coordinates are also omitted, as they are not used in any of the earlier portions of the course. Some references to sidereal time and to mean and apparent solar times are made, but students are not required to be able to convert from one of these to the others in their exercises.

Although we are able to make full use of the planetarium that is available at Kutztown University, these exercises can also be done using a celestial globe. For instance, the celestial globes manufactured by the Denoyer-Geppert Co. (5235 Ravenswood Ave., Chicago, Illinois 60640 , U.S.A.) can satisfactorily produce all of the scenarios concerning celestial sphere concepts that we demonstrate in the planetarium. Thus these exercises would also prove most useful at institutions that do not have access to a planetarium. 